\title{
Análisis predictivo del número de hemodiálisis en pacientes crónicos del departamento de Denia
}

José Luis Arroyo Vicente, Tamara Terrero Romero, Ainhoa Larumbe Rodríguez, Antonio Valdivia Pérez, Catalina Espín Abellán, Fernando Ramos Torre

\section{Servicio de Nefrología. Hospital de Dénia-Marina Salud. Alicante}

\section{Introducción:}

La población en tratamiento con hemodiálisis está aumentando rápidamente en los países industrializados, en relación con distintos factores que interaccionan de forma compleja. La elaboración de proyecciones de demanda es fundamental para planificar las necesidades de instalaciones, equipamiento y personal.

\section{Objetivo:}

Realizar una predicción del número de sesiones de hemodiálisis que se realizarán en 2013 en pacientes crónicos en el Hospital de Dénia (HdD), tanto propios como transeúntes.

\section{Material y métodos:}

Como variable resultado se emplearon el número de sesiones mensuales de hemodiálisis, tanto totales como separadas por pacientes propios y transeúntes, desde abril de 2009 a febrero de 2013. Se han tenido en cuenta las siguientes variables explicativas:

- Tendencia y estacionalidad: mes y año de la serie temporal.

- Cierre de la segunda unidad de diálisis del Departamento: se considera septiembre de 2012 como período de transición y a partir de octubre de 2012 el cierre se considera completo.

- Programa de diálisis peritoneal en el HdD: se considera operativo desde mayo de 2011.

- Ampliación de la atención a transeúntes: a partir de junio de 2012 se amplía la oferta de puestos de hemodiálisis para transeúntes, por lo que se añade esta variable explicativa al modelo. Exposición: en el caso de los pacientes propios se considera población expuesta al total de población empadronada en Denia, según datos del Instituto Valenciano de Estadística. La estimación de empadronados para los dos últimos años se ha establecido por regresión lineal simple a partir de los datos a 1 de enero de 2009, 2010 y 2011 . Se ha utilizado una regresión de Poisson para el cálculo de las predicciones en cada grupo de pacientes (propios o transeúntes).

\section{Resultados:}

El modelo predictivo resulta globalmente significativo $(p<0,001)$ y explica un $80,24 \%$ de la variabilidad observada. Tras ajuste multivariado, el año se asocia significativamente con la necesidad de hemodiálisis en la población empadronada $(p<0,001)$, observándose un incremento progresivo de la demanda. Respecto al cierre de la segunda Unidad de diálisis del Departamento, una vez pasado el periodo de transición se observa un incremento significativo $(p=0,001)$ de hemodiálisis en el HdD (RR 1,09; IC95\% 1,03-1,15). El comienzo del programa de diálisis peritoneal en el $\mathrm{HdD}$ se asocia significativamente $(p<0,001)$ con un descenso de hemodiálisis en la población empadronada (RR 0,86; IC95\% 0,82-0,91). Por medio de un modelo análogo, hemos realizado la predicción del número de diálisis en transeúntes. Al sumar las predicciones en pacientes propios y en transeúntes, obtenemos la predicción de sesiones totales de hemodiálisis en pacientes crónicos: a partir de junio de 2013 la demanda total de hemodiálisis rebasará las 1.200 sesiones al mes, alcanzando el máximo en agosto (entre 1.371 y 1.570 sesiones). 


\section{Conclusiones:}

La utilización de un modelo predictivo nos ha permitido predecir la demanda de hemodiálisis en nuestro departamento con una precisión aceptable ( $7 \%$ de diferencia entre demanda observada y predicha) y una antelación de hasta 9 meses.

Palabras clave: Dialysis, Epidemiology, Time trends, Forecasting.

\section{Referencias Bibliográficas}

1. Gilbertson DT, Liu J, Xue JL et al. Projecting the number of patients with end-stage renal disease in the United States to the year 2015. J Am Soc Nephrol (2005);16:3736-3741.

2. $P R, A m b u ̈ h l ~ M$, Corleto $D$ et al. Epidemiologic trends in chronic renal replacement therapy over forty years: a swiss dialysis experience. BMC Nephrology (2012);13:52. En: www.biomedcentral. com/1471-2369/13/52.

3. RR, Laupacis A, Hux JE et al. Forecasting the need for diálisis services in Notario, Canada to 2011. Healthcare policy (2009);4(4):e151-e161.

4. Schaubel DE, Morrison HI, Desmeules M et al. End-stage renal disease projections for Canada to 2005 using Poisson and Markov models. International Journal of Epidemiology (1998);27:274281.

5. Villa $G$, Rodríguez-Carmona A, Fernández-Ortiz $L$ et al. Cost análisis of the Spanish renal replacement therapy programme. Nephrol Dial Transplant (2011);26:3709-3714.

6. You J, Hoy W, Zhao $Y$ et al. End-stage renal disease in the Northern Territory: current and future treatment costs. MJA (2002);176:461-465. 